\title{
http://bjas.journals.ekb.eg \\ Urinary Phosphorous Excretion in Early Chronic Kidney Disease
}

A.T.Mahmoud ${ }^{1}$, M.E.Ibrahim ${ }^{1}$, A.E. Mansour ${ }^{1}$ and J.H.Sabry ${ }^{2}$

${ }^{1}$ Internal Medicine and Nephrology Dept., Faculty of Medicine, Benha Univ., Benha, Egypt

${ }^{2}$ Clinical and Chemical Pathology Dept., Faculty of Medicine, Benha Univ., Benha, Egypt

E-Mail:mahmoud22@gmail.com

\begin{abstract}
Phosphate is an essential biologic element; phosphate is required by all cells for normal function and is a critical component of all living organisms. In patient with chronic kidney disease (CKD) stages 2 and 3, the ability to excrete phosphate load is diminished.this work was aiming to detect the early changes occurring in CKD-MBD regarding the urinary phosphorous excretion This is a case-control study applied on 100 subjects admitted to Benha university hospital and Kafr shokr hospital during the period from October 2017 to December 2018.The one hundred subjects were classified into four groups:Those to start with aggregation incorporated twenty five patients with phase 1 CKD. Those second assembly included twenty five patients with phase 2 CKD. The third bunch included twenty five patients with stage $3 \mathrm{a}$ CKD. The fourth bunch incorporated twenty five Obviously solid subjects serving Likewise control. Serum phosphorous might have been 3. 39+. 52 On stage1CKD, 3. 45+. 50 On stage2CKD, Furthermore $3.845+.51$ On stage3CKD and 24h urinary phosphorous discharge might have been 714. 415+ 171. 49 Previously, stage1CKD, 587. 035+126. 23 to stage2CKD Also $429.435+141.53$ done stage3CKD. 24h urinary phosphorous discharge could make used to recognizing which tolerant for punctual phase for CKD with ordinary serum phosphorous level could profit from oral phosphate folio Also cardiovascular intercession done tolerant for CKD.
\end{abstract}

Key words: Chronic kidney disease, Serum phosphorous, 24hour urinary phosphorous excretion.

\section{Introduction}

CKD Furthermore CKD-MBD are An systemic jumble about mineral Furthermore bone digestion system showed Toward Possibly one alternately An consolidation of the following: Abnormalities about calcium, phosphorous, parathyroid hormone or vit $\mathrm{d}$ digestion system. Abnormalities for bone turnover, mineralization, volume, straight development or quality vascular alternately delicate tissue calcification [1].

Serum phosphorous level is An danger element for cardio vascular illness to tolerant with CKD. Renal phosphate taking care of will be influenced Toward phosphate stacking Also depletion, parathyroid hormone $(\mathrm{PTH}), \quad 1,25$ Di-hydroxy Vit D, hypercalcemia, hypocalcemia, glucose, corrosive build disorder, dopamine Furthermore fibroblast development variable 23 (FGF23). From claiming these PTH and FGF23 have been viewed as Similarly as particularly essential elements that control renal phosphate taking care of [2].

Developing proof demonstrates that climbing FGF23 levels clinched alongside promptly phases from claiming CKD need aid incompletely answerable for administering phosphatemia inside the ordinary extent [3].

In the promptly phases about CKD, phosphorus maintenance fortifies FGF-23 What's more PTH secretion, which thus smother renal phosphate reabsorption and expand renal phosphate discharge. FGF-23 Additionally suppresses 1,25- dihydroxyvitamin d (1,25D) production, which cutoff points intestinal phosphate absorption $\mathrm{Be}$ that permits increments clinched alongside PTH levels. While FGF-23 suppresses PTH emission done typical parathyroids safety of the impact for FGF-23 gives the idea Similarly as kidney capacity decreases due to diminished Klotho statement in the parathyroid Furthermore kidney [4]. In early $\mathrm{CKD}$, adaptive processes increase the fractional excretion of phosphate (FEpo4) and decrease the phosphate threshold clearance (TmP/GFR) to maintain normal serum phosphorous values . proximal tubule Nadependent phosphate uptake decreases in CKD as do NaPi2a mRNA and protein expression. The increase in PTH in CKD plays a central role in the adaptive process [5].

\section{Patients and method}

This will be a case-control investigation that might have been sanction by therapeutic moral Committees from claiming staff about Medicine, Benha school as stated by planet medicinal cooperation revelation from claiming helsinki 1965. The examine might have been performed at Benha school healing facility Also Kafr shokr clinic Throughout the time from october 2017 will december 2018.

\subsection{Inclusion Criteria}

CKD patients with eGFR $>45 \mathrm{ml} / \mathrm{min}$ used variable Modification of Diet in Renal Disease (MDRD) equation.

\subsection{Exclusion Criteria}

-Age $<18$ years

-Patient with active drug or alcohol dependence or abuse

-Patient with evidence of acute kidney injury or requirement for dialysis

Patient with eGFR $<45 \mathrm{ml} / \mathrm{min}$

This study was applied on One hundred persons, classified into four groups:

The first group included twenty five patients with stage 1 CKD

The second group included twenty five patients with stage 2 CKD

The third group included twenty five patients with stage 3a CKD 
The fourth group included twenty five apparently healthy subjects serving as control.

\section{Methods}

I-Medical history and clinical examination : sex, Age(years), diabetes mellitus(DM), Hypertension(HTN) and body mass index(BMI)with spotlights on cardiovascular risk factors, vital data, neck veins, peripheral pulsations and limb ischemia

\section{II-Lab investigations}

I-Biochemical tests:

Serum samples:The serum samples were used for measurement of serum urea, serum creatinine, serum calcium and serum phosphorous

- Serum samples: $4 \mathrm{ml}$ of venous blood samples were withdrawn from each patient \&control into a red capped serum separating tube. Blood was centerfugated at $3000 \mathrm{rpm}$ for $10 \mathrm{mins}$. To separate serum. Separated serum was aliquetted and kept at $20^{\circ} \mathrm{c}$ till analyzed.

Urine samples: The urine samples were used for measurement of 24hour urinary phosphorous excretion.

- Urine samples: Urine was collected for $24 \mathrm{hrs}$ starting from 8:00 am in a special container containg $10 \mathrm{mls}$ of conc $\mathrm{Hcl}$ as a preservative.

GFR estimation:
GFR was calculated according to the following equation.

$$
\mathrm{eGFR}=
$$

2- Imaging

- ECHO: for assessment of valvular calcification, left ventricular hypertrophy (LVH), regional and motion abnormalities.

\section{Statistical methods}

Measurable presentation Also examination of the exhibit study might have been conducted, utilizing those mean, standard deviation, Chi-square, straight correspondence coefficient, collector working trademark (ROC)-curve, Furthermore examination of fluctuation tests by Factual one bundle for social science (SPSS) adaptation 17. 0 (SPSS inc., Chicago, IL, USA). Chisquare theory stated that the column Also section variables are independent, without demonstrating those quality alternately course of the relationship. Those straight relationship coefficient might have been utilized for the identification for correspondence between two quantitative variables On one aggregation.

\section{Results}

Table (1) Comparison between CKD Stages regarding demographic data.

\begin{tabular}{|c|c|c|c|c|c|c|c|c|}
\hline & & & $\begin{array}{c}\text { stage } 1 \\
\text { CKD }\end{array}$ & $\begin{array}{c}\text { stage } 2 \\
\text { CKD }\end{array}$ & $\begin{array}{c}\text { stage 3a } \\
\text { CKD }\end{array}$ & $\mathrm{X} 2$ & $\begin{array}{c}P . \\
\text { value }\end{array}$ & \\
\hline Sex & $\begin{array}{c}\text { Female } \\
\text { Male }\end{array}$ & $\begin{array}{c}\text { No. } \\
\% \\
\text { No. } \\
\%\end{array}$ & $\begin{array}{c}10 \\
40.0 \% \\
15 \\
60.0 \%\end{array}$ & $\begin{array}{c}14 \\
56.0 \% \\
11 \\
44.0 \%\end{array}$ & $\begin{array}{c}11 \\
44.0 \% \\
14 \\
56.0 \%\end{array}$ & 5.649 & .130 & \\
\hline $\begin{array}{l}\text { Age } \\
\text { (years) }\end{array}$ & $\begin{array}{r}\text { Rar } \\
\text { Mean }\end{array}$ & & $\begin{array}{c}22-65 \\
40.68 \pm \\
5.64\end{array}$ & $\begin{array}{c}26-78 \\
49.82 \pm \\
4.349\end{array}$ & $\begin{array}{c}31-83 \\
56.28 \pm \\
1.44\end{array}$ & $\begin{array}{l}9.462 \\
\text { F test }\end{array}$ & .000 & $\begin{array}{l}\mathrm{P} 1=.030 \\
\mathrm{P} 2=.000 \\
\mathrm{P} 3=.077\end{array}$ \\
\hline $\begin{array}{l}\text { BM } \\
\left(\mathbf{k g} / \mathbf{m}^{2}\right)\end{array}$ & $\begin{array}{r}\text { Rar } \\
\text { Mean }\end{array}$ & & $\begin{array}{c}21-39 \\
28.68 \pm \\
2.635\end{array}$ & $\begin{array}{c}19-41 \\
27.39 \pm \\
5.235\end{array}$ & $\begin{array}{c}21-39 \\
27.209 \pm \\
5.581\end{array}$ & .47 & .62 & $\begin{array}{l}\mathrm{P} 1=.261 \\
\mathrm{P} 2=.253 \\
\mathrm{P} 3=.947\end{array}$ \\
\hline
\end{tabular}

There was no statistically significant difference between stages of CKD as regard Sex or BMI.

Table (2) Comparison between stages of CKD regarding labortory investigation.

\begin{tabular}{|c|c|c|c|c|c|c|c|}
\hline & & $\begin{array}{c}\text { stage } 1 \\
\text { CKD }\end{array}$ & stage 2 CKD & stage 3a CKD & F test & P. value & \\
\hline \multirow{2}{*}{$\begin{array}{l}\text { s.Cr } \\
(\mathrm{mg} / \mathrm{dl})\end{array}$} & Range & $.1,4-1.70$ & $1.80-2$ & $2,1-2.76$ & \multirow{2}{*}{77.553} & \multirow{2}{*}{.000} & $\mathrm{P} 1=.00$ \\
\hline & $\underset{\text { SD }}{\operatorname{Mean} \pm}$ & $\begin{array}{l}1.486 \pm \\
.12741\end{array}$ & $1.786 \pm .228$ & $2.146 \pm .568$ & & & $\begin{array}{l}\mathrm{P} 2=.00 \\
\mathrm{P} 3=.00\end{array}$ \\
\hline \multirow[b]{2}{*}{$\begin{array}{l}\text { s.Urea } \\
\text { (mg/dl) }\end{array}$} & Range & $46-69$ & $71-91$ & $93-115$ & \multirow[t]{2}{*}{60.66} & \multirow[t]{2}{*}{.00} & $\mathrm{P} 1=.00$ \\
\hline & $\begin{array}{c}\text { Mean } \pm \\
\text { SD }\end{array}$ & $\begin{array}{c}55.075 \pm \\
4.85\end{array}$ & $76.566 \pm 8.68$ & $100.345 \pm 7.31$ & & & $\begin{array}{l}\mathrm{P} 2=.00 \\
\mathrm{P} 3=.00\end{array}$ \\
\hline \multirow[b]{2}{*}{$\begin{array}{l}\text { s. Ca } \\
\text { (mg/dl) }\end{array}$} & Range & $8.48-9.36$ & $8.472-9.36$ & $8.237-9.155$ & \multirow[t]{2}{*}{4.6} & \multirow[t]{2}{*}{.82} & \\
\hline & $\begin{array}{c}\text { Mean } \pm \\
\text { SD }\end{array}$ & $\begin{array}{l}8.975 \pm \\
.022\end{array}$ & $8.801 \pm .23$ & $8.686 \pm .02$ & & & \\
\hline \multirow{2}{*}{$\begin{array}{l}\text { s.P } \\
(\mathbf{m g} / \mathbf{d l})\end{array}$} & Range & $2.80-4.50$ & $2.90-4.50$ & $2.70-5.5$ & \multirow[t]{2}{*}{.68} & \multirow[t]{2}{*}{.567} & \\
\hline & $\begin{array}{c}\text { Mean } \pm \\
\text { SD }\end{array}$ & $3.39 \pm .52$ & $3.45 \pm .50$ & $3.845 \pm .51$ & & & \\
\hline
\end{tabular}




\begin{tabular}{|c|c|c|c|c|c|c|c|}
\hline \multicolumn{8}{|c|}{ Table (2) Continue } \\
\hline glomerular & Range & $90-107.10$ & $60.80-88.90$ & $46-58.90$ & 163.29 & .00 & $\mathrm{P} 1=.00$ \\
\hline $\begin{array}{l}\text { filtration rate } \\
(\mathrm{ml} / \mathrm{min})\end{array}$ & $\begin{array}{c}\text { Mean } \pm \\
\text { SD }\end{array}$ & $\begin{array}{c}97.31 \pm \\
2.48\end{array}$ & $73.71 \pm 2.28$ & $52.16 \pm .58$ & & & $\begin{array}{l}\mathrm{P} 2=.00 \\
\mathrm{P} 3=.00\end{array}$ \\
\hline $\begin{array}{l}\text { 24h urinary } \\
\text { phosphorous }\end{array}$ & Range & $\begin{array}{l}600.12- \\
1000.20\end{array}$ & $400-900.30$ & $400-600.40$ & 23.5 & .000 & $\mathrm{P} 1=.000$ \\
\hline $\begin{array}{l}\text { excretion } \\
(\mathrm{mg} / \mathrm{d})\end{array}$ & $\begin{array}{c}\text { Mean } \pm \\
\text { SD }\end{array}$ & $\begin{array}{c}714.415 \pm \\
171.49\end{array}$ & $587.035 \pm 126.23$ & $\begin{array}{c}429.435 \pm \\
141.53\end{array}$ & & & $\begin{array}{l}\mathrm{P} 2=.000 \\
\mathrm{P} 3=.000\end{array}$ \\
\hline
\end{tabular}

There were no statistical significant differences between stages of CKD regarding serum calcium or serum phosphorous.

There were statistical significant differences between stages of CKD regarding glomerular filtration rate and $24 \mathrm{~h}$ urinary phosphorous excretion.

Table (3) Correlation between 24 hours urinary phosphorous excretion and serum phosphorus, serum calcium, glomerular filtration rate and serum creatinine.

\begin{tabular}{lcc}
\hline \multirow{2}{*}{ Correlation } & \multicolumn{2}{c}{ Pearson's correlation } \\
\cline { 2 - 3 } & $\mathbf{r}$ & $\mathbf{p}$ \\
\hline 24h urinary phosphorous excretion * s.P & $-.605-$ & .000 \\
24h urinary phosphorous excretion * $\mathbf{s}$. Ca & .639 & .000 \\
24h urinary phosphorous excretion $*$ glomerular & .922 & .000 \\
filtration rate & & \\
24h urinary phosphorous excretion $*$ s.Cr & $-.785-$ & .000 \\
\hline
\end{tabular}

There were statistically significant negative correlations between 24 hours urinary phosphorous excretion and serum phosphorus, serum creatinine.

There were statistically significant positive correlations between 24 hours urinary phosphorous excretion and serum calcium, glomerular filtration rate.

Table (4) Correlation between glomerular filtration rate and serum calcium, serum phosphorous.

\begin{tabular}{llc}
\hline \multirow{2}{*}{ Correlation } & \multicolumn{2}{c}{ Pearson's correlation } \\
\cline { 2 - 3 } & \multicolumn{2}{c}{$\mathbf{r}$} \\
\hline glomerular filtration rate $*$ s. Ca & .501 & .000 \\
glomerular filtration rate $*$ s.P & $-.538-$ & .000 \\
\hline
\end{tabular}

There were statistically significant positive correlations between glomerular filtration rate and serum calcium. There were statistically significant negative correlations between glomerular filtration rate and serum phosphorus.

Table (5) Comparison difference between stage 1 CKD, stage 2 CKD and stage 3a CKD regarding Echo finding.

\begin{tabular}{lccccccc}
\hline & & stage 1 & stage 2 & stage 3a & X2 & P.value \\
& & & CKD & CKD & CKD & P1=.04 \\
& Diastolic dysfunction & No. & 6 & 6 & 7 & $28.0 \%$ & P2=.03 \\
& & $\%$ & $24 \%$ & $24.0 \%$ & 2 & P3=.51 \\
Echo & Ischemic heart & No. & 2 & 2 & $8 \%$ & \\
finding & Disease(IHD) & $\%$ & $8 \%$ & $8 \%$ & 12 & 14.6 & .01 \\
& Lvh & No. & 8 & 6 & $48 \%$ & \\
& Systolic dysfunction & No. & 0 & $32 \%$ & $24 \%$ & 3 & \\
& & $\%$ & $0 \%$ & $8 \%$ & $12 \%$ & \\
& \multirow{2}{*}{ Normal } & No. & 9 & 8 & 0 & \\
\end{tabular}

The Echo examination of the stage $1 \mathrm{CKD}$ was $24 \%$ diastolic dysfunction, $8 \%$ IHD, $32 \%$ LVH, .0\% RHD, .0\% systolic dysfunction and $36 \%$ was normal. In stage 2 . 
CKD, The Echo examination was $24 \%$ diastolic dysfunction, $8 \%$ IHD, 24\% LVH, $4.0 \%$ RHD, $8 \%$ systolic dysfunction and $32 \%$ was normal. In stage $3 \mathrm{a}$ CKD, TheEcho examination was $28 \%$ diastolic dysfunction, $8 \% \quad$ IHD, $48 \% \quad$ LVH, $12 \%$ systolic dysfunction and $.0 \%$ was normal.

\section{Discussion}

CKD will be associated for a amount challenges that help poor tolerant outcomes, An champion around the individuals those lion's share remarkable is mineral Also bone disorder [7]. Phosphate might make essential should presence be that Similarly as its amassed Might handle pernambuco wood effects. Such example might aggravate seen secured nearby end-stage renal contamination (ESRD) patients at expansive vascular Besides fragile tissue calcifications happen as an delayed consequence of incessant phosphate amassing. Finished right on occasion when stages around CKD, serum phosphate might be consistently upheld inside those Common degree owing of the compensatory augment looking into fibroblast improvemen factor-23 (FGF-23) Additionally parathyroid hormone [8].

Agdestis secured close by our consider may have been $40,68+5,64$ to period $1 \mathrm{CKD}, 49,82+4,349$ to stage 2 CKD Also 56, 28+ 1, 44 Previously, stage3A CKD.

Period will be An fundamental predictor figure to CKD patients, Concerning illustration a huge amount challenges distinguished for period for CKD patients. Those proposed period of the ESRD patients Previously, egypt stretched beginning with 45,6 truly A percentage occasion when should 1996 around 49,8 a respectable length for run through Previously, 2008 [9] those stretching mean agdestis of the ESRD patients reflects those change from asserting medicinal benefits ,but, we might even now a long way from other made countries Concerning delineation those proposed time in the united states may need been 59,2 quite a while [10] and the Normal agdestis in the europe may bring been 60,3 quite a while [11]. Secured close by japan, the individuals mean agdestis of the entire dialysis tolerant number could bring been 66,9 quite a while [12]. Moreover, Concerning illustration stated Toward 2015 USRDS twelve-month data report out the individuals pervasiveness of approached ESKD for each million populaces might have been The greater part foremost on people time of reptiles 65-74 very a portion run through.

Secured close by our think about we uncovered serum phosphorous and serum calcium were standard to CKD stages In addition this could need been concordant with catherine et al who included that Hyperphosphatemia happens Concerning outline glomerular filtration rate declines for moved CKD this Might an opportunity should make exhibited Toward wasteful urinary phosphorous release joined together for confounded bone remodling In addition continued ingestion starting with asserting phosphate [13]. Renal phosphate dealing with may be regulated at three organs parathyroid, kidney In addition bone through three response loops. These three counter managerial loops keep up solidly controlled intestinal absorption Moreover serum phosphate centralization. Ahead punctual phase CKD, serum phosphorous may bring been demonstrated up for sit tight in the average scope due to An stretch secured nearby phosphorous release at FGF-23[14].

People with CKD might to secondary danger to CVD and the region over CKD often convolutes CVD prescription and prognosis and the rate about family who knowledge cardiovascular routines might a chance to be higher around those people with CKD In around the people without CKD [15].

CKD Moreover CVD allocation similar risk factors, a considerable measure from claiming kin from claiming which have help About distinguished for lifestyle, compelled physical activity, smoking auto auto In addition improper dietary habits, Therefore obstructed renal worth of effort may settle on recognized Also Concerning illustration An self-sufficient peril figure to headway to CVD [16].

For our ponder progressions in the heart were found around CKD patients periods 1, 2 Moreover 3, these discovering were recognized at resonation. Those an extensive parcel essential resonation discoveries around instances might have been LVH 34,7\% taken following at diastolic brokenness $25,3 \%$ likewise this could need been concordant with distinctive investigations to example, Satish etal who found Echocardiographic transforms ahead non-dialysis CKD were LVH 51,35\% diastolic brokenness $35,13 \%$ systolic brokenness $21,62 \%$ pericardial radiation $0 \%$ ischemia $21,62 \%$ conventional $24,32 \%$ [17].

Urinary release from asserting phosphorous will a chance to be a end estimation of the measure from asserting phosphorous ingested Furthermore absorbed, also that prepared to those counterbalance starting with asserting bone remodling Moreover extraosseous exchange [18].

In this analyze we ran across gradual decrease with respect to urinary phosphorous release with progressive lessening to kidney function, A percentage authers guess that enduring personal satisfaction from asserting 24hour pee $\mathrm{p}$ release could be considerably All the more loathsome done clinical act to example, Stremke et al who propose that 24-hour pee phosphorous (P) release prone reflect not best dietary phosphate yet every last one of also, transforms should $\mathrm{p}$ counterbalance to patients for CKD What's more reduced kidney fill in [19].

Adamasco et al included that 24-hour pee $p$ estimation ought on at present those relic that we have acknowledged it on be, specifically a pointer to net $p$ absorption, over steady- state states. Those rehashed estimations from asserting urinary $\mathrm{p}$ release at present remain from claiming personal satisfaction to CKD attention oversaw economy in the real-world clinical setting. An right explanation of the achieve each shortages may be needed, distinguishing that $p$ adjustment may a chance to be affected inevitably Tom's examining serum $\mathrm{P}, \mathrm{PTH}$, and the dietetic also 
pharmacological intercessions accepted in the solitary tolerant [20].

\section{Conclusion}

We support the role of $24 \mathrm{~h}$ urinary phosphorous excretion for early detection of mineral disorder in asymptomatic CKD.

\section{Reference}

[1] Kidney Disease: Improving Global Outcomes (KDIGO) CKDMBD Working Group. KDIGO clinical practice guideline for the diagnosis, evaluation, prevention, and treatment of chronic kidney disease-mineral and bone disorder (CKD-MBD). Kidney Int Suppl, Vol. 113,pp.S1S130, 2009.

[2] T.Kawasaki, Maeda. Yoshitaka, Matsuki.Hisazumi . Urinary phosphorus excretion per creatinine clearance as a prognostic marker for progression of chronic kidney disease. BMC Nephrology 2015,Vol.16,pp.116-121,2015.

[3] B. Rodrigo Oliveira, L.E. Ana Cancela, G. Fabiana Graciolli .Early Control of PTH and FGF23 in Normophosphatemic.. Clin J Am Soc Nephrol. 2010. ,Vol.5,pp.286-291,2010.

[4] K.Martin and A. Esther. Prevention and Control of Phosphate Retention/ Hyperphosphatemia in CKD-MBD: What Is Normal, When to Start, and How to Treat?. Clin J Am Soc Nephrol,Vol. 6,pp.440-446. 2011.

[5] A.Felsenfeld, S. Barton Levine, and Rodriguez. Mariano.Serum calcium and bone effect of PTH, phosphate, vitamin D and uremia. Nefrologia 34,Vol. (5),pp.658-669,2014.

[6] M.M.Wieneke, C.G.Diana, V.Marion, G.E.Elise. Performance of the Cockcroft-Gault, MDRD, and New CKD-EPI Formulas in Relation to GFR, Age, and Body SizeClin J Am Soc Nephrol,Vol. 5(6),pp.1003-1009,2010.

[7] M. Ketteler, G.J.Elder, P.Evenepoel, J.H.Ix. Revisiting KDIGO clinical practice guideline on chronic kidney disease-mineral and bone disorder: a commentary from a Kidney Disease: Improving Global Outcomes controversies conference. Kidney Int. ,Vol.87(3),pp.502-28,2015.

[8] S.Disthabanchong,. Phosphate and Cardiovascular Disease beyond Chronic Kidney Disease and Vascular Calcification Clin Chem. ,Vol. 63(2),pp.464-474,2018.

[9] A.Afifi,. The Egyptian renal registry. The 9th annual report for the year 2008. Published on 29th Annual Congress of Nephrology of Egyptian Society of Nephrology and Transplantation (ESNT)., Vol.25 (4),pp.256-261,2008.
[10] United States Renal Data System. 2015 USRDS annual data report: epidemiology of kidney disease in the United States. Bethesda, MD: National Institutes of Health, National Institute of Diabetes and Digestive and Kidney Diseases, Vol.8(2),PP.548-565, 2015.

[11] M.Pippias, V.S.Stel, J.M.Abad Diez, N.Afentakis, J.A.Herrero-Calvo , M.Arias . Renal replacement therapy in Europe: a summary of the 2012 ERAEDTA Registry Annual Report. Clin Kidney J,Vol.8,pp.248-261. 2015

[12] S.Nakai, N.Hanafusa, I.Masakane, M.Taniguchi, T.Hamano, T.Shoji. An overview of regular dialysis treatment in Japan (as of 31 December 2012). Ther Apher Dial 2014,Vol. 18,pp.535-602, 2014.

[13] M. S.Catherine, H. C.Matthew, K.Alexander and M.G.Cecilia.Arterial Calcification in Chronic Kidney Disease: Key Roles for Calcium and PhosphateCirc Res., Vol. 109(6),PP.697$711,2011$.

[14] P.Narayan, \& B. Dharmendra. Renal phosphate handling: PhysiologyIndian J Endocrinol Metab,Vol. 17(4),PP.620-627, 2013.

[15] M.Gallieni. DASH and Mediterranean Diets as Nutritional Interventions for CKD Patients by the National Kidney Foundation, Inc,Vol.12(2),pp.0272-6386,2016.

[16] C.S.Fox, K.Matsushita , M.Woodward, Associations of kidney disease measures with mortality and end-stage renal disease in individuals with and without diabetes: A metaanalysis of 1024977 individuals. Lancet,Vol. 380, pp.1662-1673,2012.

[17] S.Satis, K.Tarvinderijit, K.Satinderpal, Kamalpreet . ECG and ECHO Changes in CKD annals of international medical and dental research ISSN (0),Vol.12(9),pp.2395-2822,2017,

[18] C.Francisco, V.Juan, Elena D.V.García, M.V.Coral, M.G.Rocío Relationship between serum phosphorus and the progression of advanced chronic kidney disease DOI: 10.3265 /Nefrologia .pre 2011.Sep.11089,2011.

[19] E.R.Stremke, L.D.McCabe, G.P.McCabe, B.Martin . Twenty-four-hour urine phosphorus as a biomarker of dietary phosphorus intake and absorption in CKD. Clin J Am Soc Nephrol, Vol. 13,PP.1002-1012, 2018

[20] C.Adamasco \& G.Maurizio. Urinary Phosphorus Excretion Not What We Have Believed It to Be? Clin J Am Soc Nephrol.,Vol.13(7),PP.973$974,2018$. 
